\title{
Therapeutic Itinerary of the Patients Followed for Myasthenia Gravis in Dakar
}

\author{
Kaba Yacouba ${ }^{1,}$, , Mourabit Salaheddine ${ }^{1}$, Atsa Kouda Daniel ${ }^{1}$, Affognon Cadnelle Christelle ${ }^{1}$, \\ Bugeme Marcellin $^{1}$, Fall Maouly ${ }^{2}$, Seck Lala Bouna ${ }^{1}$, Ndiaye Moustapha ${ }^{1}$, Diop Gallo Amadou ${ }^{1}$ \\ ${ }^{1}$ Neurology Department, Faculty of Medicine, Pharmacy and Dentistry of the Sheikh Anta Diop University of Dakar, Neurosciences Clinic I. \\ P Ndiaye - Fann National University Hospital Center, Dakar-Senegal \\ ${ }^{2}$ Neurology Department, Faculty of Medicine, Pharmacy and Dentistry of the Sheikh Anta Diop University of Dakar, Pikine National \\ Hospital, Dakar-Senegal
}

Email address:

yacoukaba12gmail.com (K. Yacouba)

${ }^{*}$ Corresponding author

\section{To cite this article:}

Kaba Yacouba, Mourabit Salaheddine, Atsa Kouda Daniel, Affognon Cadnelle Christelle, Bugeme Marcellin, Fall Maouly, Seck Lala Bouna, Ndiaye Moustapha, Diop Gallo Amadou. Therapeutic Itinerary of the Patients Followed for Myasthenia Gravis in Dakar. Clinical Neurology and Neuroscience. Vol. 4, No. 1, 2020, pp. 18-23. doi: 10.11648/j.cnn.20200401.14

Received: June 19, 2019; Accepted: February 6, 2020; Published: February 14, 2020

\begin{abstract}
Introduction: Myasthenia gravis is an autoimmune disease caused by specific autoantibodies that induce dysfunction of neuromuscular transmission. The diagnostic procedure is similar regardless of the age of the subject. As in any disease, the management starts with the diagnosis, which is often unduly delayed. The purpose of our study was to evaluate the reasons for this delay. Methodology: This was a retrospective, transversal, and descriptive study on the files of myasthenic subjects followed over a period of four years (2014 to 2017) at the neuroscience clinic of the Univresity National Center of Fann and at the consultation of neurology of the Pikine National Hospital Center where a hetero-administered questionnaire was completed. Results: The study involved 18 myasthenic patients including 13 women and 5 men with a sex ratio of 2.6. The median age of the patients was 32 years [ 14 - 60 years]. The average time to patient diagnosis was 24 months. The patients had consulted on average four times before the diagnosis of myasthenia gravis. The referral hospital was the facility attended by $78 \%$ of patients and the ophthalmology service was the first service consulted by 13 patients, or $72 \%$. The median age at onset of symptoms was 28 years. Ptosis was the first symptom in $56 \%$ of the cases. Conclusion: Myasthenia gravis is a disorder little known to the public and to practitioners. The ophthalmologist was the first remedy. It would be wise for any practitioner to know the main manifestations.
\end{abstract}

Keywords: Myasthenia Gravis, Therapeutic Itinerary, Dakar

\section{Introduction}

Myasthenia gavis is an autoimmune disease caused by specific autoantibodies that induce dysfunction in neuromuscular transmission, the consequence is an excessive fatigability of the striated muscles during exertion [1]. It can occur at any age with 2 frequency peaks, the first around the third decade and the second during the sixth decade [2]. Like most autoimmune diseases, myasthenia gravis is more common in young women with a sex ratio of $3 / 2$ whereas after 60 years the gap narrows. Myasthenia gravis is marked by a clinical polymorphism, evolving with an alternation of relapses and remissions. A very serious condition in his respiratory attack, it can be life-threatening. More generally, the incidence varies from 1.7 to 21.3 per million inhabitants, depending on the study [3]. The global prevalence varies between 43 and 64 per million inhabitants [3]. The incidence was 2 and 2.6 per million inhabitants respectively in Tanzania [4] and South Africa [5]. The diagnostic approach is similar regardless of the subject's age. The electroneuromyography and the anticholinesterase therapeutic test confirm the diagnosis [6]. Unlike young subjects, the diagnosis of myasthenia gravis appears to be underestimated in the elderly with a very long diagnostic delay [7]. As in any disease, management begins with the 
diagnosis, which is often unduly delayed in myasthenia gravis; the reasons for this delay have rarely been studied [8]. Therefore, in view of this observation, we have proposed to conduct this study which has the following specific objectives:

1. Evaluate the diagnostic delay of myasthenic patients followed in the Neurosciences department at the Fann University Hospital and at the neurology consultation of the National Hospital Center of Pikine;

2. Identify the health structures and specialties consulted before the diagnosis;

3. Analyze the diagnoses mentioned and the treatments proposed before the specialized neurology consultation;

4. Appreciate the current management of myasthenic patients.

\section{Framework of Study}

We conducted our study at the Ibrahima Pierre Ndiaye neuroscience clinic at the National University Center of Fann and at the neurology consultation at the National Hospital Center of Pikine.

\section{Type and Period of Study}

It was a retrospective, transverse, and descriptive study, relating to the files of myasthenic subjects followed over a period from 2014 to 2017; at the neuroscience clinic of the National University Hospital Center of Fann and at the neurology consultation of the National Hospital Center of Pikine. The files were recruited over a period of six months.

\section{Study Population}

Selection criteria

i. Inclusion criteria:
Were included in our study the files of all the patients followed at the neuroscience clinic of the NTHC of Fann and the consultation of neurology of the NHC of Pikine for autoimmune myasthenia gravis with clinical-paraclinical confirmation.

ii. Exclusion criteria:

Incomplete records, non-consenting myasthenic patients and unreachable patients were not included in our study.

\section{Study Method}

\section{Means of investigation:}

During the recruitment period, 26 files of myasthenic patients were collected. Among them, 19 were contacted by telephone, 5 had died and 2 were unreachable. Finally, 18 patients agreed to participate in the study. We received outpatient Myasthenic patients contacted, and they were informed about the details of the study. Patients were seen every Wednesday in group of three for six weeks. During the interview, we completed the survey form heteroadministered.

This questionnaire included items detailing sociodemographic data, personal and family pathological history. It also included: the first sign that alerted the patient, the age of onset of symptoms, the duration of the course of the disease, the time taken to diagnose, the number of consultations and health facilities attended before the final diagnosis, diagnoses mentioned before that of myasthenia gravis, the paraclinical examinations carried out and the treatment received by the patient.

\section{Results}

Our study involved 18 myasthenic patients, the majority of our 13 patients $(72 \%)$ were female, with a sex ratio of 2.6 .

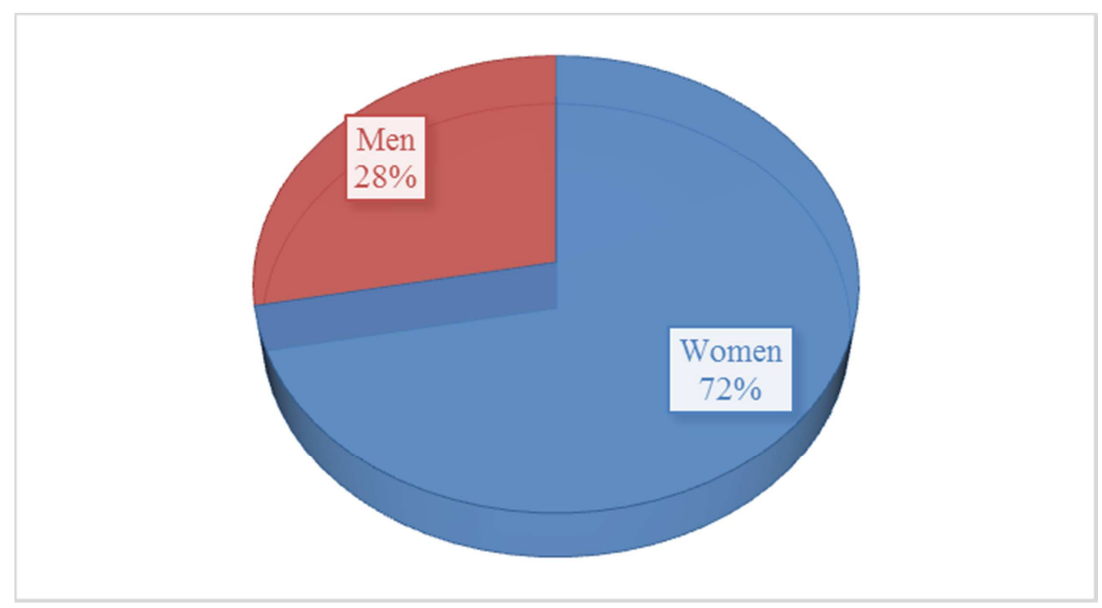

Figure 1. Distribution of patients by gender.

The median age of the patients was 32 years [14 - 60 years]. The average time to patient diagnosis was 24 months. Patients consulted on average four times before the diagnosis of myasthenia gravis.

The referral hospital was the health facility most frequented by myasthenic patients. 


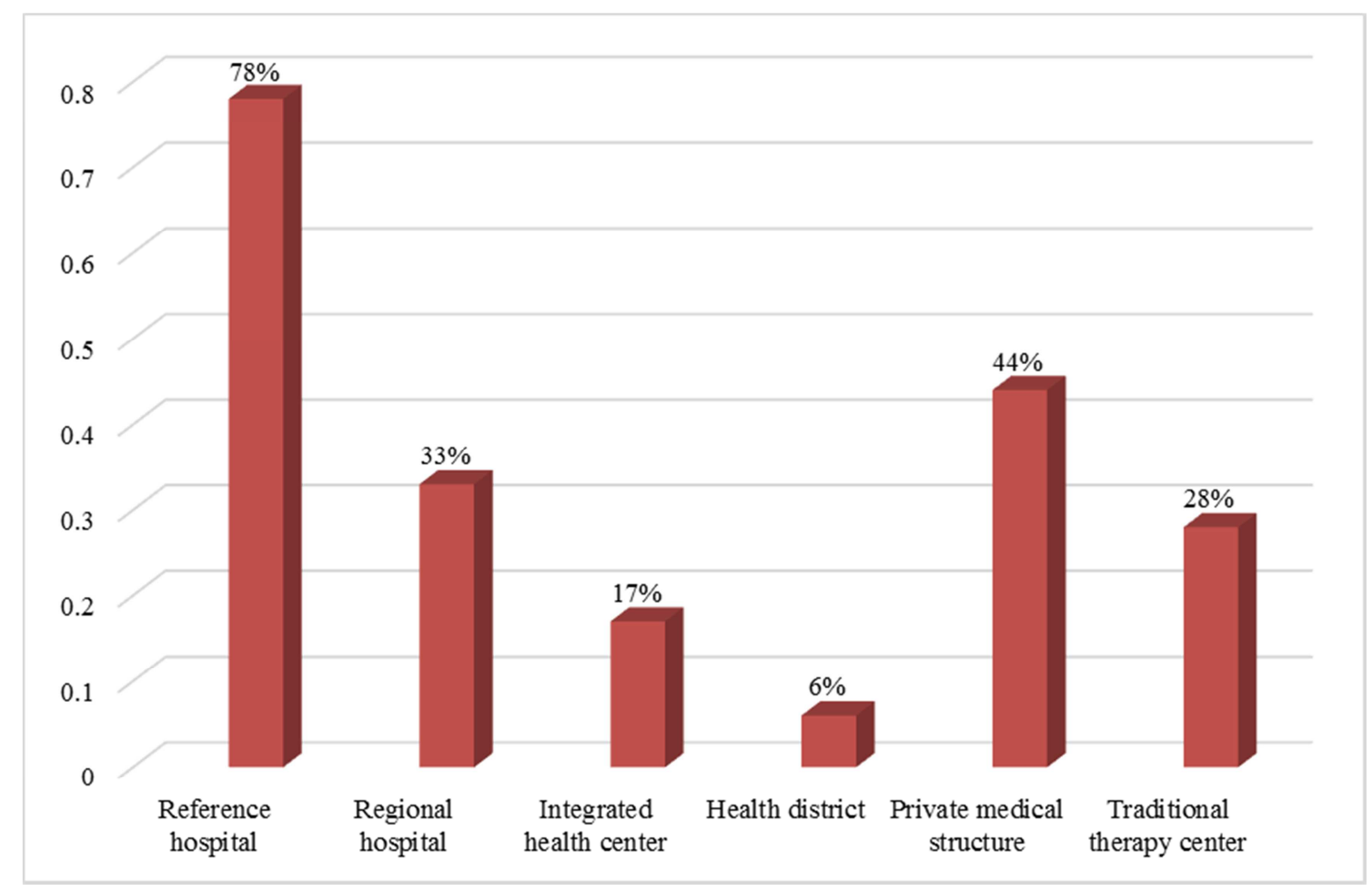

Figure 2. Distribution of patients according to the health facilities consulted.

The ophthalmology service was the first service consulted by 13 patients, or $72 \%$.

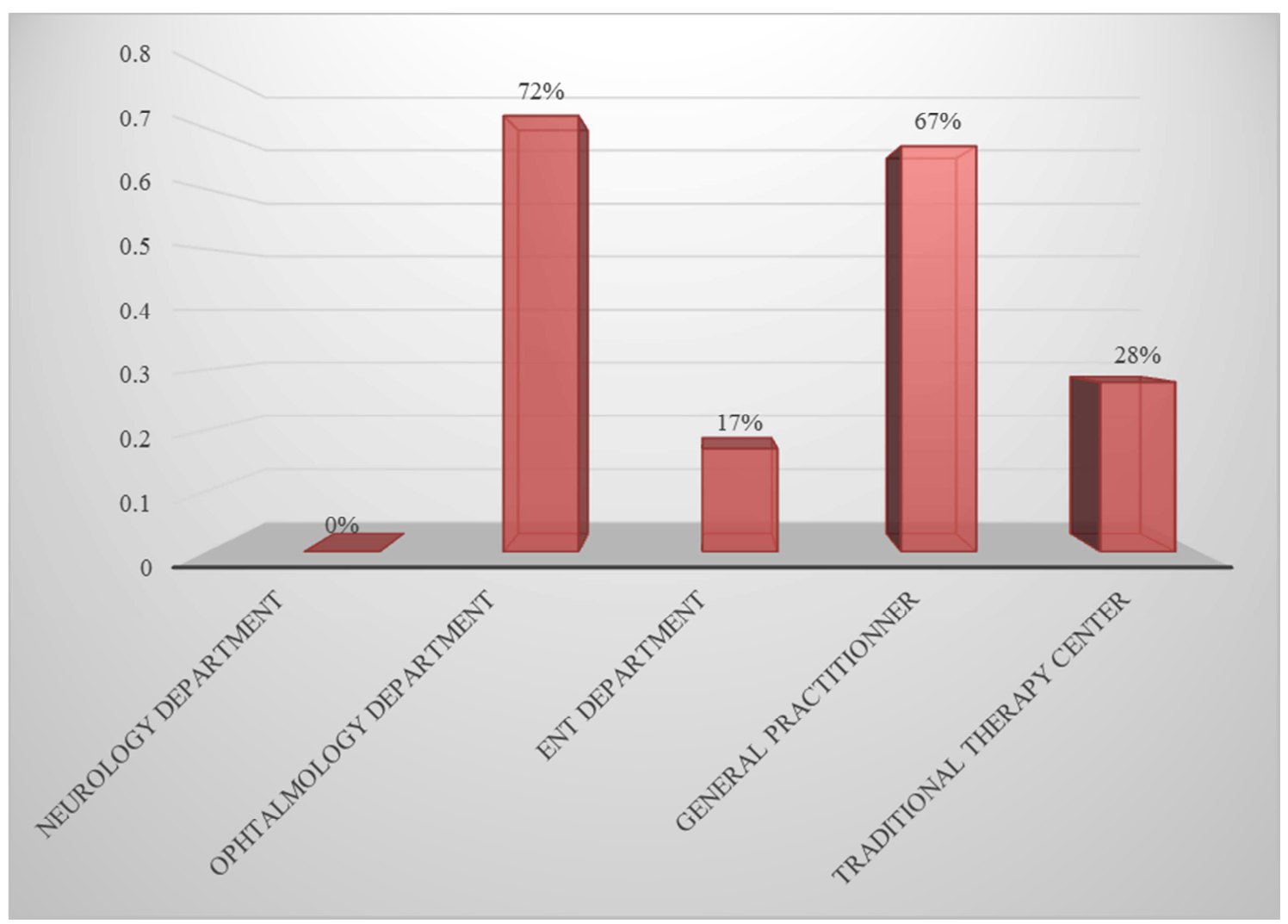

Figure 3. Distribution of patients according to the services consulted.

The median age at onset of symptoms was 28 years. Ptosis was the first symptom in $56 \%$ of the cases. The clinical signs were dominated by ocular manifestations and lesions of the lower limbs. They were found in 17 patients or $94 \%$. 
Table 1. Distribution of patients according to clinical manifestations.

\begin{tabular}{lll}
\hline Clinical Manifestations & Workforce (n) & Percentage (\%) \\
\hline Impairment (ocular + 4 limbs) & 17 & 94 \\
Phonation disorders & 12 & 67 \\
Swallowing disorders & 10 & 56 \\
Chewing deficit & 9 & 50 \\
Respiratory disorders & 5 & 28 \\
Neck extensor deficit & 3 & 17 \\
\hline
\end{tabular}

The diagnoses mentioned before that of myasthenia gravis were dominated by ophthalmological pathologies.

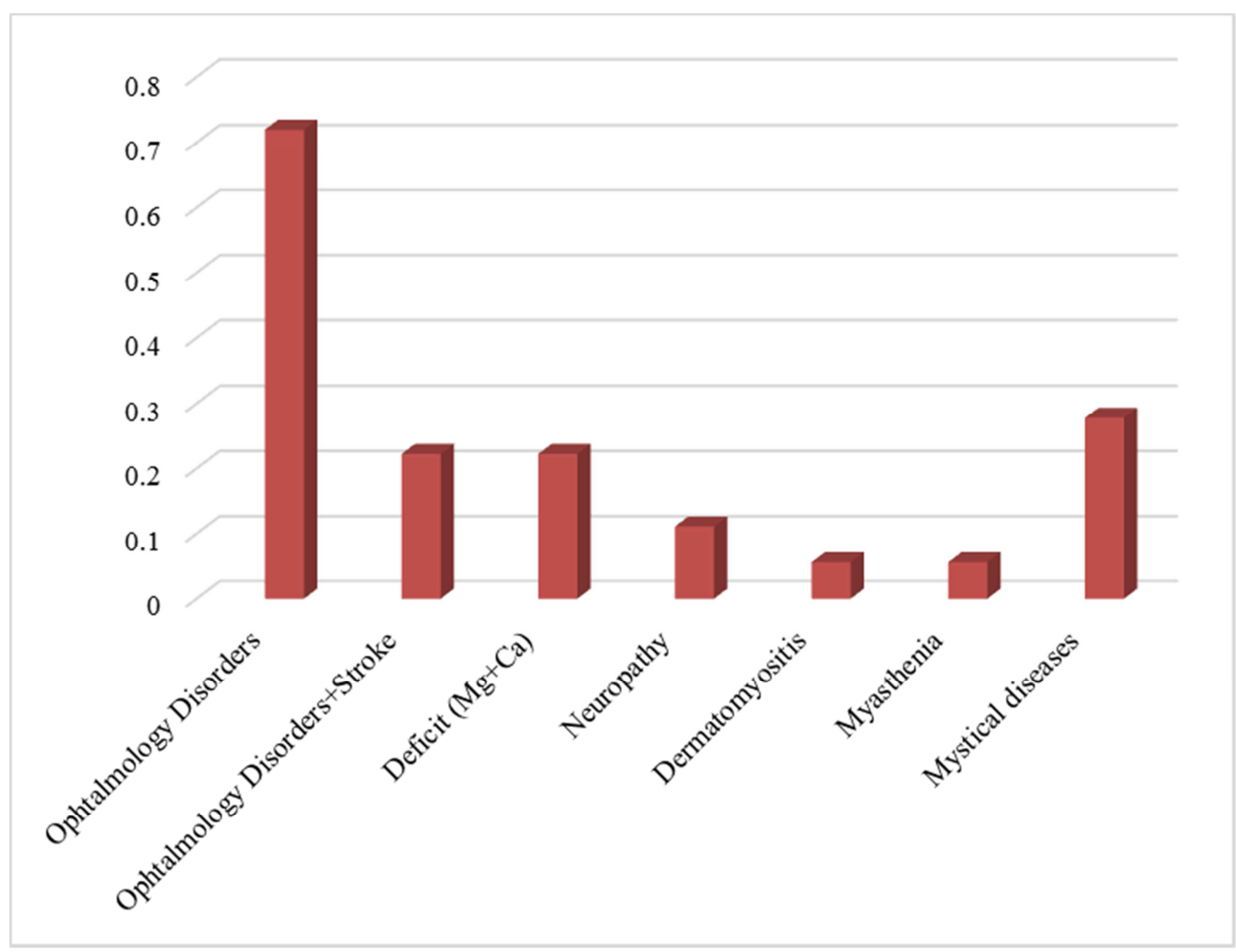

Figure 4. Distribution of patients according to the diagnoses mentioned.

Eye drops treatment was most common in myasthenic patients before diagnosis.

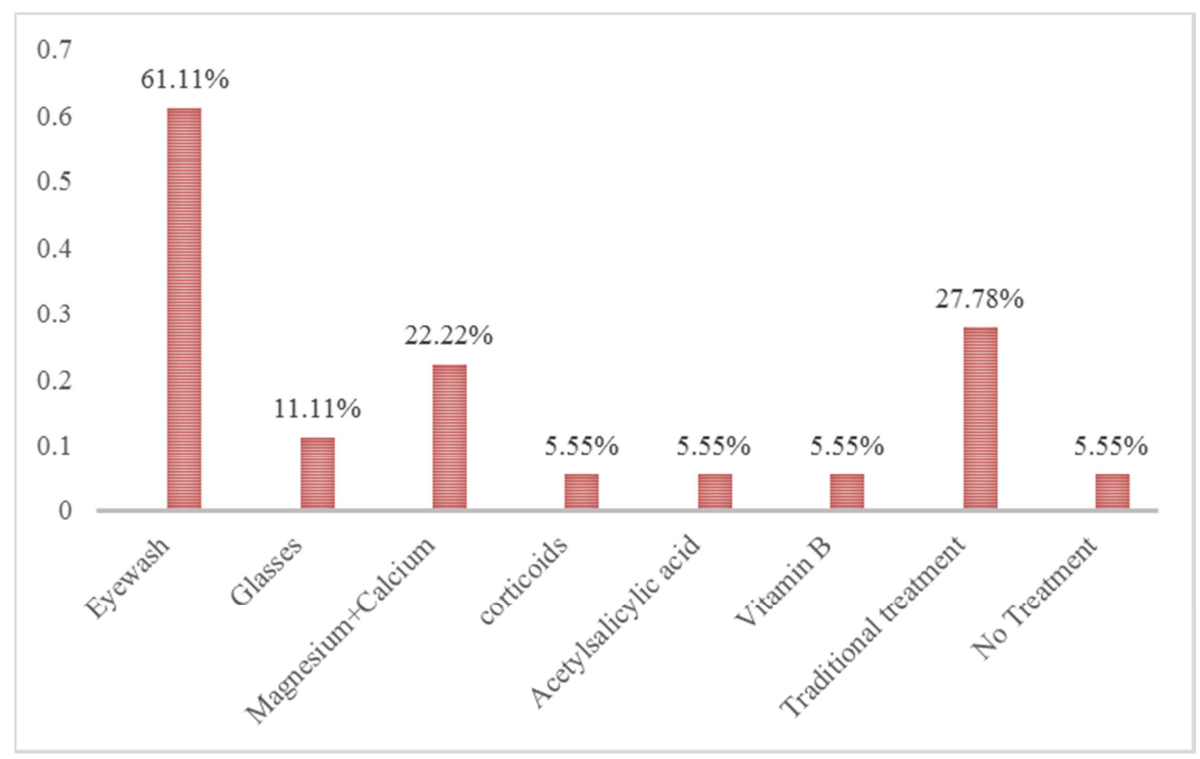

Figure 5. Treatments received before the diagnosis of myasthenia gravis. 
The anti-acetylcholine receptor antibody test was performed in all patients. The results were positive in 15 of them $(83 \%)$, negative in 2 patients and doubtful in one patient. The anti-Musk antibody test was positive in two patients.

All the patients had performed an electroneuromyography which showed a decrement.

The chest CT scan was done in $13(72 \%)$ of the patients; and 4 had a thymoma.

In terms of management, all patients were put on anticholinesterase drugs. Corticosteroids were associated with anticholinesterases in 12 patients. Immunosuppressants were associated in four (4) patients. Thymectomy was performed in three (3) patients. The course of treatment was favorable in all patients.

\section{Discussion}

Autoimmune myasthenia gravis is a relatively rare condition [1]. Its global incidence is estimated at 5.3 per million inhabitants with a variable of 1.7 to 21.3 per million inhabitants, according to studies [3]. The combined prevalence is estimated at 77.7 cases per million inhabitants [9]. This rarity was found in our study. In fact, over four years we have only received 26 cases of autoimmune myasthenia gravis. These results corroborate those of Matuja in Tanzania [10] who noted over 10 years, 47 cases of myasthenia gravis. This rarity could possibly be explained by the diagnostic error of the patients.

It is a disease that occurs at any age [1] with two frequency spikes. A first peak occurs between 20 and 40 years predominantly female [11], and a second occurs between 55 and 75 years predominantly male [12]. In $83 \%$ of cases, the disease started at a young age in our study, with a median age of onset of 27.5 years. These results are consistent with those of Holtsema in Holland [4] and Wing in Singapore [13] who reported a young onset age at 31 years respectively in 21 cases; and at 44 years in 133 cases. In our study, of the eighteen cases of myasthenia gravis that we had collected, we noted a female predominance, with 13 women for 5 men. These results are consistent with those of Wing in Singapore [13], Matuja in Tanzania [10] and Holtsema in Holland [4] who reported a sex ratio of $1.6 / 1$ respectively; $1.35 / 1 ; 2 / 1$. However, a male predominance was found in India by Singhal. Out of a cohort of 836 cases of autoimmune myasthenia gravis and a period of 43 years, there were 611 men for 225 women, ie a sex ratio of 2.7 [14].

The delay in diagnosis is significant in the elderly [7]. In our study where the disease started at a young age, the median time to patient diagnosis was 24 months. These results correspond to those of Mantegazza et al. [15] who also found a diagnostic delay of 24 months in $80 \%$ of the cases. Beekman et al. [8] reported a diagnostic delay of more than 24 months. However Lavrnić et al. reported a shorter average time to diagnosis at 11 months [16]. This difference could be explained by the small size of our sample. No simple explanation can be given for this diagnostic delay. We found in our study that patients consulted on average 4 times before the diagnosis of myasthenia gravis. The majority of these patients $(72 \%)$ saw at least twice an ophthalmologist before diagnosis. False diagnoses were raised, therefore inappropriate treatments were prescribed. Beekman et al. [8] also reported that patients consulted many specialists; expensive and non-specific tests were carried out and false diagnoses were raised.

Autoimmune myasthenia gravis has a frequently ocular onset (ptosis and / or diplopia), with extension to other muscles in $80 \%$ of cases [1]. In our study, the ocular involvement was present in all our patients, hence probably the systematic use of ophthalmological consultation. This is in accordance with the literature where ocular involvement is the most frequent manifestation [1]. Fact noted by several authors such as Zhang in the Province of Hu Bei [17] and Holtsema [4]. Ptosis was the first symptom in $56 \%$ of cases, and 17 out of 18 patients with a generalized form. Thus this fact was reported by several authors: Hyun found in a series of 71 cases of myasthenia gravis, $80 \%$ of generalized form for $20 \%$ of ocular myasthenia gravis [18]. In Wing's study of a cohort of 133 patients, $63 \%$ had a generalized form [13]. In India, Singhal noted $73.68 \%$ of generalized form [14]. However, in Zhang's study in $\mathrm{Hu}$ Bei Province, pure eye damage was more common. [17] The high frequency of ocular manifestations and the high percentage of ptosis as the first symptom in our study justify the high frequency of consultations with the ophthalmologist who rarely referred the patient to the neurologist in first line.

The diagnostic approach consists of carrying out a therapeutic anticholinesterase test, carrying out the electroneuromyography and making the determination of the antibodies, of which the anti-acetylcholine receptors are positive in $80 \%$ of the generalized forms, and $50 \%$ of the ocular forms. [2]. In our study, anti-RACh antibodies were positive in $83 \%$ of the cases of generalized forms; our results were consistent with data from the literature. Similar results were reported by several authors including Berrih [3] and Motomura [19]. However, in African Americans, less sensitivity and a lower rate of anti-RACh have been observed with a higher rate of anti MUSK in generalized forms [20].

The form with anti-Musk $\mathrm{Ab}$ represents approximately $40 \%$ of patients with a generalized form and without anti$\mathrm{RACh} \mathrm{Ab} \mathrm{[3].} \mathrm{We} \mathrm{had} \mathrm{identified} 2$ out of 18 cases of myasthenia gravis generalized with anti-Musk $+\mathrm{Ab}$, these results are consistent with the data in the literature. It should be noted that the assay of anti-Musk Ab was not carried out in patients who presented anti-RACh $+\mathrm{Ab}$.

Electroneuromyography using repetitive low frequency stimulation was performed in all our patients and found a significant decrease (greater than 10\%).

Autoimmune myasthenia gravis is associated in $20 \%$ of cases with a thymoma [1], the removal of which improves the course of the disease. In our study, unfortunately $30 \%$ of patients had not performed a chest CT scan. Of the $13(70 \%)$ 
who had done the chest CT, 4 had a thymoma.

Anticholinesterases are prescribed on the first line and may or may not be combined with other therapies. They are the basis of symptomatic treatment [21]. Our patients were specifically put on Pyridostigmine which is the most widely used and better tolerant anticholinesterase than Neostigmine [22]. Corticosteroids were associated with anticholinesterases in $2 / 3$ of the cases. Corticosteroid therapy is the oldest and most widely used of the background treatments used in myasthenia gravis. Its efficacy is widely accepted, despite the few published controlled studies [23]. Thus in four large retrospective studies on corticosteroid therapy in generalized myasthenia, more than $73 \%$ of marked improvement or even remission was noted among the 422 patients treated [22]. The use of immunosuppressants (cyclophosphamide) was observed in 4 of our patients who had not responded to corticosteroid therapy. Cyclophosphamide has been shown to be effective in refractory or severe forms of myasthenia gravis [23]. Overall, the course of treatment was favorable except in 1 patient who had progressed to death.

\section{Conclusion}

Myasthenia gravis, like other autoimmune diseases, is a complex pathology. It is marked by a clinical and paraclinical polymorphism with therapeutic difficulties. It is a rare and little-known affection of the public and practitioners. His diagnosis is often unduly delayed.

Eye damage was the main manifestation, the ophthalmologist was the first remedy.

Despite the small number of patients in our series, certain remarks seem to us to be relevant. It would be wise for any practitioner to know the main manifestations. This would make it possible to: make an early diagnosis, limit the prescription of sometimes aggravating drugs and ask the right therapeutic indications.

\section{References}

[1] B. Eymard. La myasthénie pour l'interniste. Revue Neurologie. 2014; A200-02.

[2] A. El Midaoui, O. Messouak, M. F. Belahsen. La myasthénie. Amether. 2010; 2 (1): 54-65.

[3] S. Berrih-Aknin, R. Le Panse. Myasthénie et auto-anticorps: physiopathologie des différentes entités. La Revue de médecine interne. 2014; 35: 413-420.

[4] H. Holtsema, J. Mourik, R. E. Rico, J. R. Falconi, J. B. M. Kuks, H. J. G. H. Oosterhuis. Myasthenia gravis on the Dutch Antilles: an epidemiological study. Clinical Neurology and Neurosurgery. 2001; 102 (4): 195-8.

[5] K. J. Bateman et al. Incidence of seropositive myasthenia gravis in Cape Town and South Africa. S Afr Med J. 2007; 97 (10): 959-62.
[6] A. Saraux-Bonneton, B. Raffin, F. Vautier, J. Laudet. Myasthénie et personnes âgées. À partir d'une observation. Rev Méd Interne. 2001; 22: 984-7.

[7] F. Durand, J. P. Camdessanché, L. Jomir, J. C. Antoine, P. Cathébras. Myasthénie du sujet âgé: analyse rétrospective de 23 observations. Revue Médecine interne. 2005; 26: 924-930.

[8] R. Beekman, J. B. M. Kuks, H. J. G. M. Oosterhuis. Myasthenia gravis: Diagnostic and follow-up of 100 consecutive patients. J. Neurol. 1997; 244: 112-8.

[9] A. S. Carr et al. Systematic review of population based epidemiological studies in Myasthenia Gravis. BMC Neurol. 2010; 10: 46.

[10] W. B. P. Matuja et al. Incidence and characteristics of myasthenia gravis in Dar Es Salaam, Tanzania. East African Medical Journal. 2001; 78 (9): 473-76.

[11] M. A. Hellmann, R. Mosberg-galili, I. Steiner. Myasthenia gravis in the elderly. J. Neurol Sci. 2013; 325: 1-5.

[12] A. Meyer, Y. Levy. Chapter 33: Geoepidemiology of myasthenia gravis. Autoimmunity Reviews. 2010; 9: A38386.

[13] A. U. Wing Lok et al. MMed FAMS. Myasthenia gravis in Singapore. Neurol J Southeast Asia. 2003; 8: 35-40.

[14] B. S. Singhal et al. Myasthenia gravis: A study from India. Neurol India. 2008; 56 (3): 352-55.

[15] R. Mantegazza et al. Myasthénie grave: données épidémiologiques et facteurs pronostiques. Am. Acad. Sciences de NY. 2003; 998 (1): 413-23.

[16] D. Lavrnic et al. Epidemiological and clinical characteristics of myasthenia gravis in Belgrade Yugoslavia. Acta Neurol Scand. 1999; 100: 168-174.

[17] X. Zhang et al. Clinical and serological study of myasthenia gravis in HuBei Provi China. J Neurol Neurosurg Psychiatry. 2007; 78 (4): 386-90.

[18] H. S. Roh, S. Lee, J. S. Yoon. Comparaison of clinical manifestations between patients with ocular myasthenia gravis and generalized myasthenia gravis. Korean J Ophthalmol. 2011; 25 (1): 1-7.

[19] R. Ohta, M. Motomura, B. Rinsho. Autoantibodies detected in acetylcholine receptor antibody-negative myasthenia gravis. Rinsho Byori. 2014; 62 (3): 255-260.

[20] S. J. OH et al. Racial differences in myasthenia gravis in Alabama. Muscle Nerve. 2009 Mar; 39 (3): 328-332.

[21] M. Garcia-carrasco et al. Therapeutic options in autoimmune myasthenia gravis. Autoimmunity Reviews. 2007; 6: 373-378.

[22] M. Meriggioli, D. Sanders. Autoimmune myasthenia gravis: emerging clinical and biological heterogeneity. Lancet Neurol. 2009; 8: 475-88.

[23] C. Schneider-Gold et al. Corticosteroids for myasthenia gravis. Cochrane Database Syst Rev. 2005 (2). 